\title{
Association of the G403A polymorphism in the RANTES gene with coronary artery disease: a meta-analysis
}

\author{
K. Cui, X.Y. Ge and H.L. Ma \\ Department of Epidemiology and Biostatistics, School of Public Health, \\ Liaoning Medical University, Jinzhou, Liaoning, China \\ Corresponding author: H.L. Ma \\ E-mail: mahonglinly@163.com
}

Genet. Mol. Res. 12 (3): 3912-3918 (2013)

Received November 5, 2012

Accepted February 1, 2013

Published September 23, 2013

DOI http://dx.doi.org/10.4238/2013.September.23.10

\begin{abstract}
The G403A polymorphism in the RANTES (regulated on activation normal $\mathrm{T}$ cell expressed and secreted) gene has a key role in the expression of RANTES, which has been detected in a range of cells in atherosclerotic plaque. However, the association of this polymorphism with the risk of coronary artery disease (CAD) remains controversial. A meta-analysis was performed to assess the association of the G403A polymorphism in the RANTES gene with the risk of CAD. A comprehensive search was conducted to identify all studies published on the association of the RANTES gene G403A polymorphism with CAD risk. The fixed or random-effect pooled measure was adopted based on a heterogeneity test among studies, which was evaluated using $I^{2}$. Potential sources of between-study heterogeneity were explored using meta-regression analysis. Publication bias was estimated with Begg's rank correlation method. Eight articles were included in this meta-analysis, with 4601 CAD cases and 2522 controls. No significant association of RANTES gene G403A polymorphism with CAD was identified in any of the codominant, dominant, recessive, homozygote, or heterozygote inheritance models. No evidence of publication bias
\end{abstract}


was detected. The meta-analysis suggested that the A allele of the G403A polymorphism in the RANTES gene has no effect on the risk of CAD. This relationship needs to be confirmed by further studies.

Key words: Coronary artery disease; Meta-analysis; Polymorphism

\section{INTRODUCTION}

Increasing evidence has demonstrated that chemokines play an important role in the development of atherosclerosis (AS), which is the pathogenesis of coronary artery disease (CAD) (Um and Kim, 2009). RANTES (regulation on activation normal T cell expressed and secreted), also known as C-C ligand 5, belongs to the CC chemokine family (Appay et al., 2001), and its expression has been detected in T cells in atherosclerotic plaques as well as in macrophages, endothelial cells, lymphocytes, and vascular smooth muscle cells, suggesting its role in the development and progression of AS (Szalai et al., 2001; Tavakkoly-Bazzaz et al., 2011). RANTES is coded by the RANTES gene, which is localized to the region q11.2-q12 of chromosome 17. Recently, a $\mathrm{G}$ to A transition at position -403 of the RANTES gene has been identified in the promoter region (Hajeer et al., 1999; Berg et al., 2009). This mutation affects the promoter activity of RANTES, suggesting that the single-nucleotide polymorphism (SNP) had an important role in RANTES expression (Liu et al., 1999; Nickel et al., 2000). However, the effect of this polymorphism on CAD risk is still controversial. Simeoni et al. (2004) have suggested that the RANTES -403A allele increases genetic susceptibility to CAD, whereas Jang et al. (2007) have proposed that the allele is associated with reduced CAD risk (Jang et al., 2007). Hence, in the present study, we conducted a meta-analysis with aiming to assess the effect of the RANTES G403A polymorphism on CAD risk. We also evaluated the potential heterogeneity among studies as well as potential publication bias.

\section{MATERIAL AND METHODS}

\section{Search strategy}

A search was conducted for relevant available articles published in English or Chinese from 4 databases- PubMed, China National Knowledge Infrastructure, Database of Chinese Scientific and Technical Periodicals (also known as VIP), and China Biology Medical Literature Database. The keywords used in searching were the following: coronary heart disease, ischemic heart disease, myocardial infarction, coronary artery disease or angina pectoris and RANTES, CCL5 or rs2107538 and polymorphism mut* or varia*. The bibliographies of relevant studies as well as those of relevant review articles were also examined to identify studies not captured by the search strategy.

\section{Inclusion criteria}

The CAD cases in this meta-analysis were defined as coronary heart disease, coronary artery disease (CAD), myocardial infarction, acute myocardial infarction, or angina pectoris. All identified studies were reviewed independently by two investigators to determine whether 
an individual study was eligible for this meta-analysis. The inclusion criteria were as follows: 1) case-control or cohort study published as an original study to evaluate the association between RANTES G403A polymorphism and risk of CAD; 2) numbers in case (exposed) and control (unexposed) groups for each genotype, or available data with which to calculate the numbers, were reported; and 3) both case (exposed) and control (unexposed) groups were unrelated and came from the same temporally and geographically defined underlying population. If disagreement arose between the 2 investigators about the eligibility of an article, consensus was sought with a 3rd reviewer.

\section{Data extraction}

Data were independently extracted by 2 investigators, and a consensus was reached on all of the items. To assure quality, the most recent and complete articles were chosen if a study was published more than once. The information extracted from each study was as follows: name of first author, year of publication, country, ethnic origin of the studied population, definition of cases, numbers in case (exposed) and control (unexposed) groups, genotype and allele distributions, mean age, male sex percentage in case (exposed) and control (unexposed) groups.

\section{Statistical analysis}

Chi-square analysis was used to test departure from Hardy-Weinberg equilibrium (HWE) for the genotype distribution of RANTES G403A polymorphism in control groups. The pooled measure was calculated as the inverse-variance weighted mean of the logarithm of odds ratio with $95 \%$ confidence interval for dominant (AA+AG vs GG), recessive (AA $v s \mathrm{AG}+\mathrm{GG})$, codominant (A $v s \mathrm{G})$, homozygote (AA $v s \mathrm{GG}$ ), and heterozygote (AG $v s \mathrm{GG})$ models, respectively. $I^{2}$ described the proportion of total variation attributable to betweenstudy heterogeneity as opposed to random error or chance. In the presence of substantial heterogeneity $\left(I^{2}>50 \%\right)$, the DerSimonian and Laird random-effect model was adopted as the pooling method; otherwise, the inverse-variance fixed-effect model was used. Potentially important sources of between-study heterogeneity were explored using meta-regression with restricted maximum likelihood estimation. Publication bias was estimated using Begg's rank correlation method. An analysis of influence was conducted to describe how robust the pooled estimator was to the removal of individual studies. A publication was suspected of excessive influence if the point estimate of its omitted analysis lay outside the $95 \%$ confidence interval of the combined analysis. All statistical analyses were performed with STATA version 9.2 (Stata Corporation, College Station, TX, USA). All reported probabilities (P values) were 2-sided, with $\mathrm{P}<0.05$ considered statistically significant.

\section{RESULTS}

\section{Characteristics of studies}

The search of the 4 databases identified 8 publications eligible for this meta-analysis on the relationship between RANTES G403A polymorphism and CAD; 7 of the articles were published in English and 1 was published in Chinese. These 8 articles were case-control designs that 
included $4601 \mathrm{CAD}$ cases and 2522 controls. General characteristics and the allele and genotype distributions in the published articles included in this meta-analysis are shown in Table 1.

\begin{tabular}{|c|c|c|c|c|c|c|c|c|c|}
\hline \multirow[t]{2}{*}{ Author } & \multirow[t]{2}{*}{ Year } & \multirow[t]{2}{*}{ Country } & \multirow[t]{2}{*}{ Disease } & \multicolumn{2}{|c|}{ Genotypes (AA/AG/GG) } & \multirow[t]{2}{*}{ Matched } & \multirow{2}{*}{$\begin{array}{c}\% \text { of male } \\
\text { (Case/control) }\end{array}$} & \multirow{2}{*}{$\begin{array}{c}\text { Mean age } \\
\text { (Case/control) }\end{array}$} & \multirow[t]{2}{*}{$\mathrm{P}$ for HWE } \\
\hline & & & & Case & Control & & & & \\
\hline Shi & 2010 & China & CHD & $110 / 201 / 169$ & $83 / 223 / 174$ & Age, sex & $0.72 / 0.72$ & $60.9 / 60.7$ & 0.43 \\
\hline Simeoni & 2004 & Germany & CAD & $86 / 706 / 1439$ & $23 / 137 / 370$ & None & $0.74 / 0.51$ & $63.8 / 56.9$ & 0.034 \\
\hline Tereshchenko & 2011 & Czech+Russia & MI & $22 / 135 / 310$ & $12 / 101 / 224$ & None & $0.76 / 0.52$ & $55.8 / 40.3$ & 0.88 \\
\hline Vogiatzi & 2009 & Greece & CAD & $13 / 83 / 96$ & $1 / 65 / 83$ & Age & $0.89 / 0.71$ & $64.0 / 62.0$ & 0.002 \\
\hline Park & 2007 & Korea & AMI & $17 / 70 / 82$ & $24 / 67 / 71$ & Age & $0.76 / 0.67$ & $62.6 / 62.2$ & 0.22 \\
\hline Szalai & 2001 & Hungary & CAD & 13/106/199 & 6/93/221 & Age, sex & $0.76 / 0.75$ & $57.6 / 58.9$ & 0.3 \\
\hline Tavakkoly-Bazzaz & 2011 & Iran & CAD & $14 / 61 / 116$ & $5 / 49 / 74$ & None & $0.60 / 0.69$ & $63.0 / 55.0$ & 0.37 \\
\hline Jang & 2007 & Korea & CAD & $82 / 242 / 229$ & $70 / 211 / 135$ & Age & $1.00 / 1.00$ & $54.8 / 53.8$ & 0.42 \\
\hline
\end{tabular}

HWE $=$ Hardy-Weinberg equilibrium in control group. CHD = coronary heart disease; CAD = coronary artery disease; $\mathrm{MI}=$ myocardial infarction; $\mathrm{AMI}=$ acute myocardial infarction.

\section{Quantitative synthesis}

Results of the pooled analysis are shown in Table 2. In this study, we found no statistically significant relationship between the RANTES gene G403A polymorphism and CAD in any of the 5 inheritance models. Strong evidence for heterogeneity among studies was detected in 5 models, and the pooled results from the random-effect model were appropriate for drawing the conclusion. Two studies were excluded for deviation from HWE in controls, and the pooled measure was meta-analyzed repeatedly in each of the 5 inheritance models. $I^{2}$ was altered but still higher than $50 \%$ in all of the models except for the heterozygote model.

Table 2. Pooled measures on the association between RANTES gene G403A polymorphism and CAD.

\begin{tabular}{llccc}
\hline Data & Inherited model & FEM pooled OR $(95 \% \mathrm{CI})$ & REM pooled OR $(95 \% \mathrm{CI})$ & $I^{2}(\%)$ \\
\hline All relevant articles & Dominant & $1.012(0.979-1.047)$ & $1.002(0.934-1.073)$ & 60.4 \\
& Recessive & $1.055(0.986-1.128)$ & $1.145(0.970-1.350)$ & $1.022(0.968-1.078)$ \\
& Codominant & $1.018(0.990-1.047)$ & $1.129(0.949-1.343)$ & 83.3 \\
& Homozygote & $1.031(0.962-1.106)$ & $0.985(0.916-1.060)$ & 52.9 \\
& Heterozygote & $1.004(0.969-1.041)$ & $0.976(0.891-1.068)$ & 56.8 \\
Excluded for DHWE & Dominant & $0.972(0.917-1.030)$ & $1.097(0.953-1.264)$ & 54.7 \\
& Recessive & $1.064(0.974-1.163)$ & $1.005(0.929-1.086)$ & 64.5 \\
& Codominant & $0.999(0.955-1.044)$ & $1.070(0.902-1.268)$ & 65.5 \\
& Homozygote & $1.023(0.930-1.124)$ & $0.955(0.880-1.036)$ \\
\hline
\end{tabular}

Dominant model: AA+AG vs GG; recessive model: AA vs AG+GG; codominant model: A vs G; Homozygote model: AA vs GG; heterozygote model: AG vs GG; DHWE: deviated from HWE in controls.

However, the aforementioned association patterns were not appreciably altered.

\section{Exploration of the sources of heterogeneity}

After excluding 2 studies in which the controls deviated from HWE, univariate metaregression analysis was performed in each inheritance model. The covariates included publi- 
cation year, ethnicity (categorized as Asian and non-Asian), sex (ratio of male percent in cases to that in controls), age (ratio of mean age in cases to that in controls), and sample size. As shown in Table 3, none of the above-mentioned covariates was identified as a potential source of between-study heterogeneity in any of the 5 models.

Table 3. Meta-regression analysis for exploration of the sources of heterogeneity.
\begin{tabular}{lccccc}
\hline Inherited model & \multicolumn{7}{c}{ P value for the covariate in univariate meta-regression } \\
\cline { 2 - 5 } & Ethnicity & Year & Age & Sex & Sample size \\
\hline Dominant & 0.173 & 0.435 & 0.989 & 0.875 & 0.881 \\
Recessive & 0.360 & 0.765 & 0.895 & 0.728 & 0.904 \\
Codominant & 0.268 & 0.564 & 0.962 & 0.929 & 0.892 \\
Homozygote & 0.255 & 0.627 & 0.898 & 0.820 & 0.878 \\
Heterozygote & 0.113 & 0.334 & 0.999 & 0.717 & 0.685 \\
\hline
\end{tabular}

Dominant model: AA+AG vs GG; recessive model: AA vs AG+GG; codominant model: A $v s$ G; homozygote model: AA vs GG; heterozygote model: AG vs GG.

\section{Influence analysis}

After excluding 2 articles that deviated from HWE in controls, the analyses of influence demonstrated that no individual study had excessive influence on the pooled effect in any of the above-mentioned inheritance models with regard to CAD (data not shown).

\section{Publication bias evaluation}

After excluding 2 articles that deviated from HWE in controls, no statistically significant publication bias was detected in any of the above-mentioned inheritance models (data not shown).

\section{DISCUSSION}

In a study by Simeoni et al. (2004), 3316 individuals were subdivided into a CAD group or a control group based on coronary angiography, and they were genotyped. Their results demonstrated that the RANTES -403A allele was associated with CAD independent of conventional risk factors. By contrast, Jang et al. (2007) have suggested that the -403A allele was associated with lower serum RANTES concentration and described the significant protective effect of the polymorphism on CAD risk in Korean male patients with or without type 2 diabetes (Jang et al., 2007). Most of the studies in this meta-analysis showed no relationship between the polymorphism and CAD risk (Szalai et al., 2001; Park et al., 2007; Vogiatzi et al., 2009; Shi Ying et al., 2010; Tavakkoly-Bazzaz, et al., 2011; Tereshchenko et al., 2011).

Because the inconsistent results from relatively small studies are underpowered for detecting polymorphism effects, a meta-analysis is an appropriate tool through which to obtain a more definitive conclusion about the role of the polymorphism in CAD risk. Our metaanalysis of 8 published articles including $4601 \mathrm{CAD}$ cases and 2522 controls from different ethnicities afforded a greater possibility to reach reasonably strong conclusions. Similar to other findings, the results of our study indicated that the RANTES G403A polymorphism was unassociated with CAD risk. 
Our meta-analysis showed significant between-study heterogeneity in all 5 inheritance models. An undetermined number of characteristics that varied among studies could have caused the heterogeneity. Thus, meta-regression was adopted to reduce between-study heterogeneity and explore its potential sources using covariates including publication year, ethnicity, sex, age, and sample size. However, after excluding 2 studies that deviated from HWE in controls, the results did not identify any of the above-mentioned covariates as an important source of heterogeneity. Given the small number of publications included in the present study, other possibilities related to disease-effect diversity cannot be ruled out, such as variations in design quality and genotyping, even though no significant individual study influenced the pooled effect and no publication bias was observed. In addition, a study has demonstrated that the G403A and another polymorphism of the RANTES gene, In1.1T/C (a C for T substitution in intron 1), were in strong linkage disequilibrium (An et al., 2002), whereas the association of an SNP genotype with a specific phenotype might be due to linkage disequilibrium with a truly functional locus (Boger et al., 2005). The genetic susceptibility may also depend on the coincidence of several gene polymorphisms acting together (Hoidal, 2001).

In conclusion, the present meta-analysis suggested that the G403A polymorphism in the RANTES gene had no association with the risk of CAD. Because potential biases, confounders, and linkages among various SNPs cannot be completely ruled out in this study, further investigation is needed to confirm these results.

\section{REFERENCES}

An P, Nelson GW, Wang L and Donfield S (2002). Modulating influence on hiv/aids by interacting rantes gene variants. Proc. Natl. Acad. Sci. U. S. A. 99: 10002-10007.

Appay V and Rowland-Jones SL (2001). RANTES: a versatile and controversial chemokine. Trends Immunol. 22: 83-87.

Berg KK, Madsen HO, Garred P, Wiseth R, et al. (2009). The additive contribution from inflammatory genetic markers on the severity of cardiovascular disease. Scand. J. Immunol. 69: 36-42.

Boger CA, Fischereder M, Deinzer M, Aslanidis C, et al. (2005). RANTES gene polymorphisms predict all-cause and cardiac mortality in type 2 diabetes mellitus hemodialysis patients. Atherosclerosis 183: 121-129.

Hajeer AH, al Sharif F and Ollier WE (1999). A polymorphism at position -403 in the human rantes promoter. Eur. J. Immunogenet. 26: 375-376.

Hoidal JR (2001). Genetics of COPD: present and future. J. Eur. Respir. 18: 741-743.

Jang Y, Chae JS, Hyun YJ and Koh SJ (2007). The rantes -403g >a promoter polymorphism in korean men: Association with serum rantes concentration and coronary artery disease. Clin. Sci. 113: 349-356.

Liu H, Chao D, Nakayama EE, Taguchi H, et al. (1999). Polymorphism in rantes chemokine promoter affects hiv-1 disease progression. Proc. Natl. Acad. Sci. U. S. A. 96: 4581-4585.

Nickel RG, Casolaro V, Wahn U, Beyer K, et al. (2000). Atopic dermatitis is associated with a functional mutation in the promoter of the C-C chemokine RANTES. J. Immunol. 164: 1612-1616.

Park S, Youn JC, Shin DJ, Park CM, et al. (2007). Genetic polymorphism in the pregnancy-associated plasma protein-A associated with acute myocardial infarction. Coron. Artery Dis. 18: 417-422.

Shi Y, Peng YD, Bao XQ and He MA (2010). The associationof g403a polymorphism in rantes gene promoter region with coronary artery disease. The J. Practical Med. 777-779.

Simeoni E, Winkelmann BR, Hoffmann MM, Fleury S, et al. (2004). Association of RANTES G-403A gene polymorphism with increased risk of coronary arteriosclerosis. J. Eur. Heart. 25: 1438-1446.

Szalai C, Duba J, Prohaszka Z, Kalina A, et al. (2001). Involvement of polymorphisms in the chemokine system in the susceptibility for coronary artery disease (CAD). Coincidence of elevated Lp(a) and MCP-1 -2518 G/G genotype in CAD patients. Atherosclerosis 158: 233-239.

Tavakkoly-Bazzaz J, Amiri P, Tajmir-Riahi M and Javidi D (2011). Rantes gene mrna expression and its -403 g/a promoter polymorphism in coronary artery disease. Gene 487: 103-106.

Tereshchenko IP, Petrkova J, Voevoda MI, Taborsky M, et al. (2011). CCL5/RANTES gene polymorphisms in Slavonic patients with myocardial infarction. Mediators Inflamm. 2011: 525691. 
Um JY and Kim HM (2009). Polymorphisms of RANTES and IL-4 genes in cerebral infarction. J. Mol. Neurosci. 37: 1-5. Vogiatzi K, Voudris V, Apostolakis S, Kochiadakis GE, et al. (2009). Genetic diversity of RANTES gene promoter and susceptibility to coronary artery disease and restenosis after percutaneous coronary intervention. Thromb. Res. 124: 84-89. 